\title{
FENOLOGÍA DE CUATRO ESPECIES DE CACTÁCEAS DEL CERRO UMARCATA. VALLE DEL RÍO CHILLÓN. CANTA-LIMA. PERÚ
}

\section{PHENOLOGY OF FOUR SPECIES OF CACTI AT UMARCATA HILL. CHILLÓN. LIMA. PERU}

\author{
Natali Ramírez-Bullón ${ }^{1}$, Aldo Ceroni-Stuva² y Viviana Castro-Cepero²
}

\begin{abstract}
Resumen
Se estudiaron cuatro especies de cactáceas: Esposta melanostele, Haageocereus pseudomelanostele subsp. aureispinus, Melocactus peruvianus y Neoraimondia arequipensis subsp. roseiflora, presentes en el ecosistema árido Cerro Umarcata, Valle del Río Chillón, CantaLima. Se encontraron dos fenofases, floración y fructificación con ocho estadios fenológicos entre Octubre 2006 y Setiembre 2007. Para la fenofase de floración, los estadios fenológicos Flor Marchita y Botón Abortado fueron dominantes, mientras que para la fenofase de fructificación, el estadio fenológico Fruto Maduro se presentó en un porcentaje bajo durante el año. El factor Temperatura se correlacionó positivamente con los estadios fenológicos Flor Marchita, Fruto Apareciendo, Fruto Maduro y Fruto Dehiscente, mientras que el factor Humedad Relativa se correlacionó positivamente con los estadios fenológicos Botón Floral, Floración Total, Fruto Apareciendo y Fruto Verde.
\end{abstract}

Palabras clave: Cactaceae, fenología, fenofases, estadio fenológico.

\begin{abstract}
Four species of cacti found in the Cerro Umarcata, Chillon River Valley, Canta-Lima arid ecosystem were studied. Phenological patterns for flowering and fruiting were recorded for Espostoa melanostele, Haageocereus pseudomelanostele subsp. aureispinus, Melocactus peruvianus and Neoraimondia arequipensis subsp. roseiflora between October 2006 and September 2007. Eight phenological stages were identified. For flowering, "Wilted Flower" and "Aborted Flower Bud" were the most common phenological stages. For the fruiting phenophase "Mature Fruit" was observed at low frequency during the study. Temperature was positively correlated with the frequency of "Wilted Flower", "Developing Fruit", "Mature Fruit" and "Dehiscent Fruit". Relative Humidity was positively correlated with the "Flowering Bud", "Open Flower", "Developing Fruit" and "Immature Fruit". phenological stages

Key words: Cactaceae, phenology, phenological stages.
\end{abstract}

\section{Introducción.}

En los ecosistemas áridos de los valles Lima se desarrollan con frecuencia las cactáceas columnares, formaciones vegetales dominantes, donde las diferentes especies juegan un rol vital en el flujo de energía como eje principal de todos los procesos y en la cadena trófica de una serie de organismos, al constituir la cobertura vegetal dominante (Ceroni \& Castro, 2006; Ceroni et al., 2007). Dentro de los ecosistemas áridos en Lima, el cerro Umarcata, en el Valle del Río Chillón, se presenta como una zona de alta diversidad específica de cactáceas (Teixeira et al., 2004). En este ecosistema están representadas 2 subfamilias, 8 géneros y un total de 9 especies. En la subfamilia Cactoideae se encontraron Neoraimondia arequipensis subsp. roseiflora, Espostoa melanostele, Haageocereus pseudomelanostele subsp. aureispinus, Haageocereus acranthus, Cleistocactus acanthurus subsp. faustianus, Melocactus peruvianus y Mila nealeana; mientras que en la subfamilia Opuntioideae, Austrocylindropuntia pachypus y Cumulopuntia sphaerica (Castro, 2006). Estudios realizados en esta zona en cuanto a la taxonomía, diversidad y densidad de las cactáceas (Castro, 2006 y Teixeira et al., 2004), del rol de la especie dominante Neoraimondia arequipensis subsp. roseiflora como eje de las interacciones en ecosistemas áridos (Novoa et al., 2003; 2005a; 2005b, 2005c y Ceroni et al., 2007), así como de conservación ex situ de cactáceas de Lima (Ceroni et al., 2004), demuestran que el cerro Umarcata es un área importante a conservar no sólo por ser sede de una alta diversidad de especies, algunas endémicas para el Perú, sino también por los futur os trabajos de investigación que puedan hacerse a fin de entender más la dinámica para una mejor 
gestión de los ecosistemas áridos del Perú (Ceroni \& Castro, 2006).

La fenología es el estudio de los fenómenos biológicos acomodados a cierto ritmo periódico, como la brotación, la florescencia y la maduración de los frutos. Como es natural, estos fenómenos se relacionan con el clima de la localidad en que ocurren. De la fenología se pueden interpretar consecuencias relativas al clima y, sobre todo al microclima, en especial ni uno ni otro se conoce debidamente. Font Quer (2000) señala que: “... la fenología de una especie depende de su propia idiosincrasia y del ciclo de dinamismo del medio, sobre todo y más generalmente del ciclo climático". Los estados fenológicos de una especie pueden afectar a la planta a múltiples niveles, incluyendo los sucesos reproductivos individuales de la planta, interacciones con otros organismos (afectando la variación y abundancia espacio-temporal de polinizadores y dispersores), dinámicas poblacionales vegetales y el funcionamiento del ecosistema (Bronstein, 1995; McIntosh, 2002).

Las bases de datos fenológicos están siendo cada vez más solicitadas por investigadores de diferentes disciplinas en biología. Las bases de datos históricas permiten establecer calendarios fenológicos que indican los cambios biológicos a través del tiempo. El uso de los datos aerobiológicos permite obtener datos diarios numéricos sobre el contenido diario e incluso horario de polen en el aire. Esta información complementa a la que se obtiene a partir de datos fenológicos de campo, que se suelen tomar una o dos veces a la semana provocando que las diferentes fases fenológicas sean estimadas por interpolación lineal para ofrecer los datos diarios (Galán, 2006). Tanto las variables meteorológicas como las feno-climáticas se suelen utilizar para ajustar los modelos fenológicos locales, con la posibilidad de obtener modelos y predicciones a nivel regional (Galán, 2006). Conocer la dinámica de la floración y fructificación es imprescindible cuando se quiera propiciar el manejo sustentable de sus recursos, más aún, cuando éstos se encuentran sometidos a un proceso de degradación por las actividades antrópicas (Rondón \& Pulido, 2001).

Entre los estudios de fenología en el Perú destacan el de Novoa et al., (2005), el primero en la zona de estudio (Umarcata), realizado con el cactus Neoraimondia arequipensis subsp. roseiflora y el de Laura (2007), sobre la fenología reproductiva de la familia Cactaceae en la Comunidad Campesina de Atiquipa (Caravelí, Arequipa-Perú). A nivel internacional, destaca el de Colaço et al., (2006) que comprende la fenología, biología floral, polinización y sistema de apareamiento de dos especies de Melocactus, y el de Ruiz et al. (2000) realizado con tres cactáceas columnares y una cactácea decumbente, en el enclave seco interandino de La Tatacoa, Colombia. Así mismo, sobre el estudio de la fenología reproductiva de tres especies simpátricas de cactus columnares, Stenocereus griseus, Pilosocereus repandus y Pilosocereus lanuginosus en la isla de Curaçao (Petit, 2001) y el de fenología floral en dos especies de cactus: Ferocactus cylindraceus y Ferocactus wislizenii en Tuczon, Arizona (McIntosh, 2002). Por otra parte, la fenología de cactáceas ha sido ampliamente considerada en otros procesos ecológicos tales como la polinización por murciélagos y polillas, así como la dispersión de semillas por murciélagos (Sahley, 1996; Nassar, et al., 1997; Valiente et al., 1996, 1997; Fleming \& Holland, 1998; Rojas et al., 1999; Holland \& Fleming, 1999, 2002; De Viana et al., 2001; Molina et al., 2004, Ibarra- Cerdeña et al., 2005).

En el presente trabajo se reporta la fenología de cuatro especies de cactáceas del cerro Umarcata: $E$. melanostele, $H$. pseudomelanostele subsp. aureispinus, $M$. peruvianus y $N$. arequipensis subsp. roseiflora, así como la correlación de las fenofases presentes con la temperatura y la humedad relativa (HR).

\section{Materiales y métodos.}

Ubicación.

El área de investigación es el cerro Umarcata, el cual está ubicado en la cuenca baja del río Chillón, a la altura del kilómetro 69 de la carretera Lima-Canta, en el distrito de Santa Rosa de Quives, provincia de Canta, departamento de Lima. Se encuentra a una altitud de 1,260 msnm y a $11^{\circ} 37^{\prime} 39^{\prime \prime}$ Latitud Sur y $76^{\circ} 46^{\prime} 9^{\prime}$ ' Longitud Oeste (Figura 1).

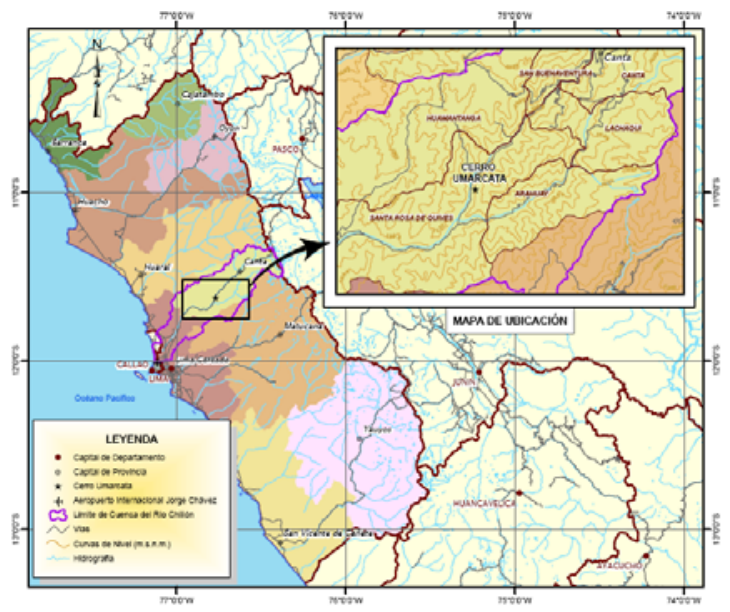

Figura 1. Ubicación del área de estudio.

Vegetación en la zona de vida de la cuenca del río Chillón.

La cuenca baja del río Chillón está conformada por una sola zona de vida correspondiente al Matorral Desértico Subtropical (md-ST) el cual se ubica entre los 800 y 2100 msnm. Abarca un área de $2030 \mathrm{~km}^{2}$. El clima es árido y semicálido. La precipitación 
Ecol. apl. Vol. 13 № 2, pp. 177-185

promedio anual es mayor a los $125 \mathrm{~mm}$ y la temperatura promedio anual es $18^{\circ} \mathrm{C}$. La vegetación natural está constituida en la parte más baja por un piso de cactáceas columnares y otra vegetación reducida. El monte ribereño está formado por Salix humboldtiana, Acacia macracantha, Baccharis salicifolia, Arundo donax, Schinus molle y Caesalpinia spinosa. En el piso de cactáceas predominan: Neoraimondia arequipensis, Cereus amazonicus, Calymmanthium sp., Melocactus sp., Jatropha macrantha, Orthopterygium huancui y Viguiera sp. Estas plantas aumentan su densidad hacia los 1000 msnm (ONERN, 1976; Weberbauer, 1945). Determinación del estado fenológico.

Se identificaron 3 estratos altitudinales y en cada uno de ellos se realizó un transecto de $100 \mathrm{~m}$ de largo. En estos se marcaron 3 individuos al azar, teniendo en total 9 individuos por especie. Se realizaron visitas mensuales desde octubre del 2006 hasta setiembre del 2007. Se evaluaron los estadios fenológicos presentes en las diferentes épocas del año siguiendo la escala de Campbell (1970) citado por Rondón (1994) modificada para estas especies, considerando a los botones abortados como un estadio fenológico por presentarse en gran cantidad (Petit, 2001; McIntosh, 2002) (Tabla 1). Los datos fueron registrados cuantitativamente indicando el número de estructuras observadas en cada estadio fenológico. Se construyeron fenogramas (Rondón, 1994) para ilustrar los estadios fenológicos en el tiempo.

Tabla 1. Fenofases y estadios fenológicos.

\begin{tabular}{cl}
\hline \multicolumn{1}{c}{ Fenofase } & Estadio Fenológico \\
\hline Floración & Botón Floral \\
& Botón \\
& Abortado \\
& Floración \\
& Total \\
& Flor \\
& Marchita \\
Fructificación & Fruto \\
& Apareciendo \\
& Fruto \\
& Verdes \\
& Fruto \\
& Maduros \\
& Fruto \\
& Dehiscente \\
\hline
\end{tabular}

Definición de los estadios fenológicos.

- Botón floral: yema floral, capullo.

- Botón abortado: yema floral marchita (sin turgencia, flácido, o seco).

- Floración total: flor totalmente abierta.

- Flor marchita: flor sin turgencia, seca.

- Fruto apareciendo: ovario desarrollado con flor marchita presente.
- Fruto verde: fruto inmaduro que presenta el pericarpo grueso de color verde, o dependiendo de la especie, granate. Generalmente de menor tamaño que el fruto maduro.

- Fruto maduro: presenta el pericarpo grueso de color rojizo o verde según la especie, pero de consistencia carnosa turgente.

- Fruto dehiscente: fruto que presenta aberturas hechas por herbívoros o agentes de dispersión.

Correlación de la fenología con los factores medio ambientales.

Durante las evaluaciones de campo, desde octubre del 2006 hasta setiembre del 2007, se registró la temperatura y humedad relativa con ayuda de un termohigrómetro con el propósito de correlacionar las diferentes fases fenológicas con estos parámetros meteorológicos. El análisis se realizó con una prueba de ordenación multivariada (Correlación Canónica). Antes de aplicar esta prueba, los datos fueron normalizados a través de una transformación raíz cuadrada de $\mathrm{x}+1$. Para el análisis de datos se utilizó el software PAST versión 1.84.

\section{Resultados y discusión.}

Espostoa melanostele (Vaupel) Borg

El estadio Flor Marchita fue el más abundante. El $64.9 \%$ de los individuos evaluados se encontraron en este estadio durante todo el año. Este estadio fenológico presentó 2 máximos en los meses de julio y octubre, cada uno con 9 individuos y con 29 a 83 flores, respectivamente. El estadio Floración Total fue observado con el $1.7 \%$ en un individuo en los meses de abril y junio con 2 y 1 flor, respectivamente. Sólo se observó un individuo con un fruto maduro en el mes de septiembre lo que representó el $0.8 \%$ del total de registros. El estadio Fruto Dehiscente representó el $16.2 \%$ entre los meses de julio a octubre, cuyo máximo de registros fue en el mes de julio con 5 individuos en este estadio y un total de 12 frutos observados (Figuras 2, 3 y 4).

Haageocereus pseudomelanostele subsp. aureispinus (Rauh \& Backeberg) Ostolaza

El estadio Flor Marchita fue el más abundante. El $25.4 \%$ de los individuos evaluados se encontraron en este estadio durante todo el año. Este estadio presentó el máximo, en el mes de setiembre, con 7 individuos y 28 flores marchitas. El estadio Floración Total se presentó con el 3.1\% de los registros en los meses de julio, agosto, setiembre y octubre, alcanzando el máximo en el mes de setiembre con 6 flores en 2 individuos evaluados. El estadio Fruto Maduro se presentó con el $4.3 \%$ del total de registros, en un individuo marcado en los meses abril, junio, julio, setiembre, octubre, noviembre y diciembre, cuyo mayor número de frutos fue 4 en el mes de setiembre (Figuras 5, 6 y 7). 


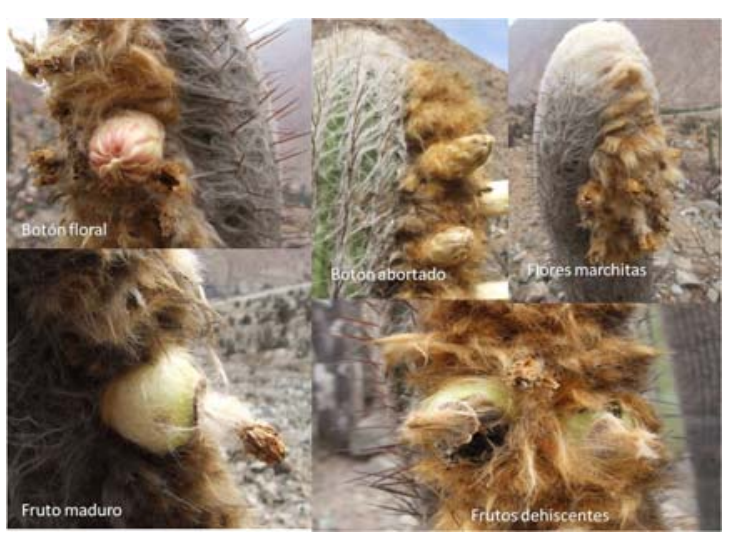

Figura 2. Estadios fenológicos para las fenofases de floración y fructificación de Espostoa melanostele (Vaupel) Borg. Cerro Umarcata. Valle del río Chillón.

Melocactus peruvianus Vaupel

El estadio Flor Marchita fue el más abundante. El 49.7\% de los individuos evaluados se encontraron en este estadio durante todo el año y fue observado en 8 individuos entre los meses febrero a agosto y en el mes de octubre. En los meses de setiembre, noviembre y diciembre fueron registrados 9 individuos en este estadio. El máximo número de flores se registró en los meses de marzo y diciembre con 187 y 230 flores, respectivamente. El estadio Floración Total, con el $14.2 \%$ de registros, se presenta en todos los meses del año, con 2 máximos de 4 individuos cada uno en los meses de marzo y octubre con 12 y 10 flores, respectivamente. El estadio Fruto Maduro se presentó con $8.1 \%$ de registros en los meses de febrero, abril, julio, agosto, setiembre, octubre, noviembre $\mathrm{y}$ diciembre, con el máximo de producción de frutos en los meses de agosto $y$ diciembre con 5 frutos en los 3 individuos registrados (Figuras 8, $9 \mathrm{y}$ 10).

Neoraimondia arequipensis subsp. roseiflora (Wedermann \& Backeberg) Ostolaza

El estadio Botón Abortado fue el más abundante. El 26.3\% de los individuos evaluados se encontraron en este estadio con 9 individuos durante todo el año, presentando el mayor número de botones abortados en los meses de agosto y setiembre con 396 y 395 botones, respectivamente. El estadio Floración Total con $2.6 \%$ de los registros se presentó con el máximo en el mes de marzo presentando 9 flores en 3 individuos evaluados. El estadio Fruto Maduro con $5.5 \%$ de los registros se presentó en los meses de febrero, marzo, abril, mayo, junio, setiembre, octubre, noviembre y diciembre, alcanzando 2 máximos de producción de frutos en los meses de mayo con 22 frutos en 5 individuos y en el mes de octubre con 21 frutos en 3 individuos (Figuras 11, 12 y 13).

En base a los resultados de fenología se puede ver que los estadios dominantes para las cuatro especies evaluadas corresponden a Flor Marchita y Botón Abortado, mientras que Floración Total y Fruto Maduro se presentan en porcentaje bajo durante el año. Sin embargo, es posible establecer épocas de colección de flores y frutos:

Espostoa melanostele: flores completamente desarrolladas (floración total) en abril y junio y frutos maduros en los meses de julio y agosto.

Haageocereus pseudomelanostele subsp. aureispinus: flores desarrolladas y frutos maduros en el mes de setiembre.

Melocactus peruvianus: flores desarrolladas en los meses de marzo y abril, y frutos maduros en agosto, setiembre y diciembre.

Neoraimondia arequipensis subsp. roseiflora: flores completamente desarrolladas en los meses de marzo y abril. Dichas fechas coinciden con el estudio de fenología de Novoa (2005) en que el mes de abril correspondió al estadio Floración Total. Así mismo, los mejores meses para obtener frutos maduros corresponden a mayo y octubre. Este resultado difiere del estudio de Novoa (2005) (Octubre 2003-Setiembre 2004) que determinó que el mes de agosto es el más adecuado para obtener frutos maduros. Por otro lado,

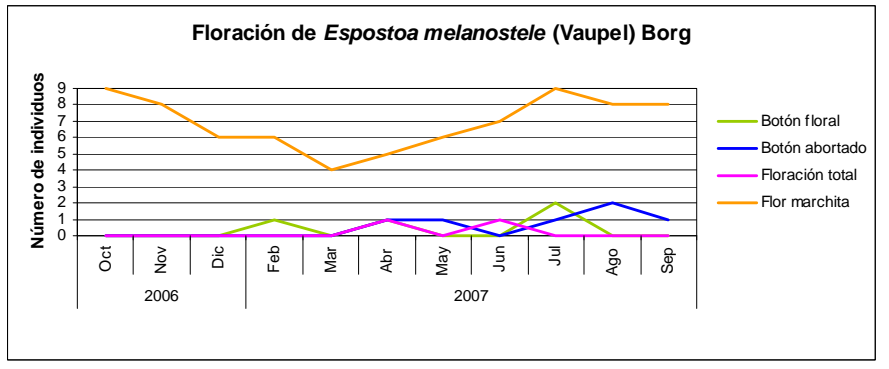

Figura 3. Fenograma de las etapas de floración de Espostoa melanostele (Vaupel) Borg, en relación con el número de individuos Octubre 2006 - Setiembre 2007.

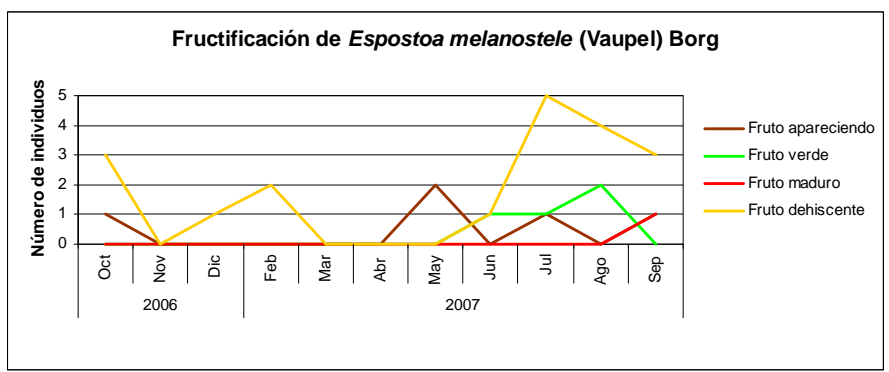

Figura 4. Fenograma de las etapas de fructificación de Espostoa melanostele (Vaupel) Borg, en relación con el número de individuos Octubre 2006 - Setiembre 2007. 


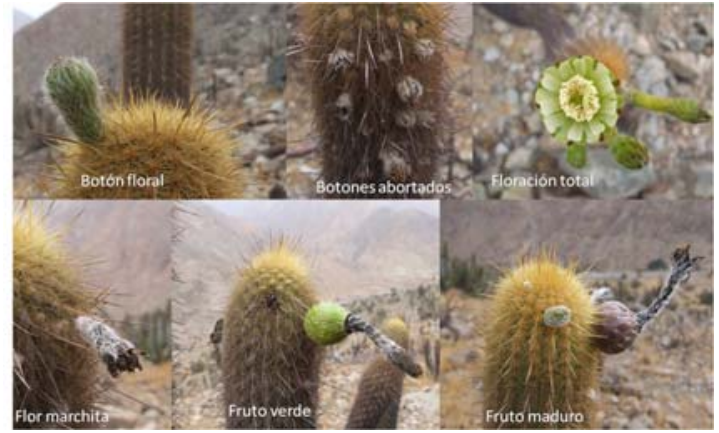

Figura 5. Estadios fenológicos para las fenofases de floración y fructificación de Haageocereus pseudomelanostele subsp. aureispinus (Rauh \& Backeberg) Ostolaza. Cerro Umarcata. Valle del río Chillón.

el estadio fenológico más abundante fue Botón Abortado con $26.3 \%$ del número de registros seguido por los estadios Botón Floral con 25\% y Fruto Verde o Inmaduro con 13.5\%. Novoa (2005) encontró 8 estadios fenológicos de los cuales el estadio Botón Floral fue el más abundante presentándose en casi el 60\% de las plantas durante los meses de octubre 2003 hasta setiembre 2004, seguido por los estadios Fruto Verde o Inmaduro con 35.36\% y Botón Abortado con 32.78\%.

Correlación Canónica de los factores medio ambientales $\left(\mathrm{T}^{\circ} \mathrm{C}\right.$ y HR) y los estadios fenológicos

Espostoa melanostele (Vaupel) Borg

Los resultados indican que la temperatura es un parámetro ambiental de significancia para los estadios fenológicos Fruto Apareciendo y Flor Marchita. En los meses de octubre, noviembre y diciembre se presentaron los mayores valores de temperatura lo que aumentó el número de flores marchitas. El estadio fenológico Fruto Apareciendo presentó una tendencia similar a la temperatura. Sin embargo, la HR presentó sus mayores valores en los meses de marzo y abril lo que favoreció la Floración Total, Botón Floral e inversamente proporcional al estadio Botón Abortado (Figura 14).

Haageocereus pseudomelanostele subsp. aureispinus (Rauh \& Backeberg) Ostolaza

Se observa que la temperatura es un parámetro ambiental de significancia para el estadio fenológico Fruto Apareciendo. En los meses de setiembre, octubre y noviembre presentaron mayores valores de temperatura lo que favoreció a este estadio. Sin embargo, la HR presentó una relación inversamente proporcional al estadio fenológico Flor Marchita, cuyos valores menores se presentaron en los meses de setiembre y octubre lo que favoreció al mayor número de flores marchitas (Figura 15).

Melocactus peruvianus Vaupel

Los resultados muestran que la temperatura es un parámetro ambiental de significancia para el estadio fenológico Fruto Apareciendo. En los meses de setiembre y octubre se presentaron mayores valores de temperatura lo que favoreció a este estadio. Sin embargo, la HR presentó sus mayores valores en los meses de febrero y marzo lo que favoreció al estadio Floración Total. El estadio Flor Marchita es inversamente proporcional a la HR (Figura 16).

Neoraimondia arequipensis subsp. roseiflora (Wedermann \& Backeberg) Ostolaza

Se observa que la temperatura es un parámetro ambiental de significancia para los estadios fenológicos Fruto Maduro y Fruto Dehiscente. Sin

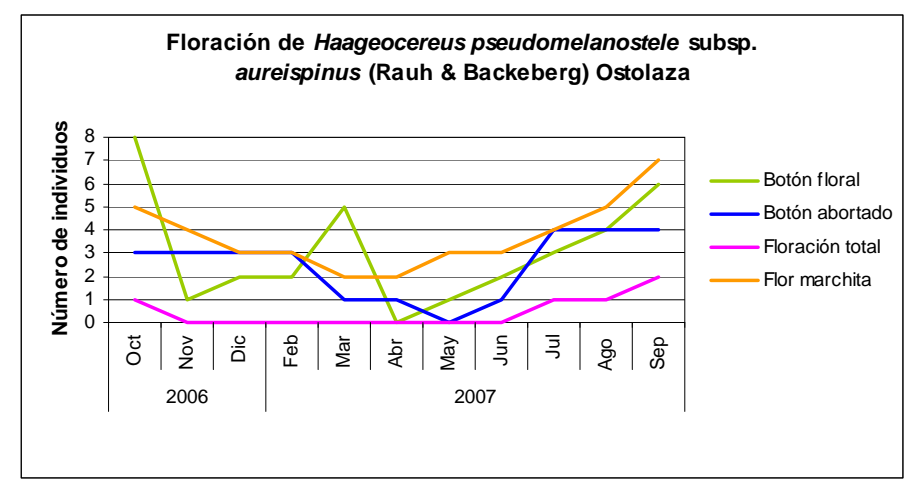

Figura 6. Fenograma de las etapas de floración de Haageocereus pseudomelanostele subsp. aureispinus (Rauh \& Backeberg) Ostolaza, en relación con el número de individuos Octubre 2006 - Setiembre 2007.

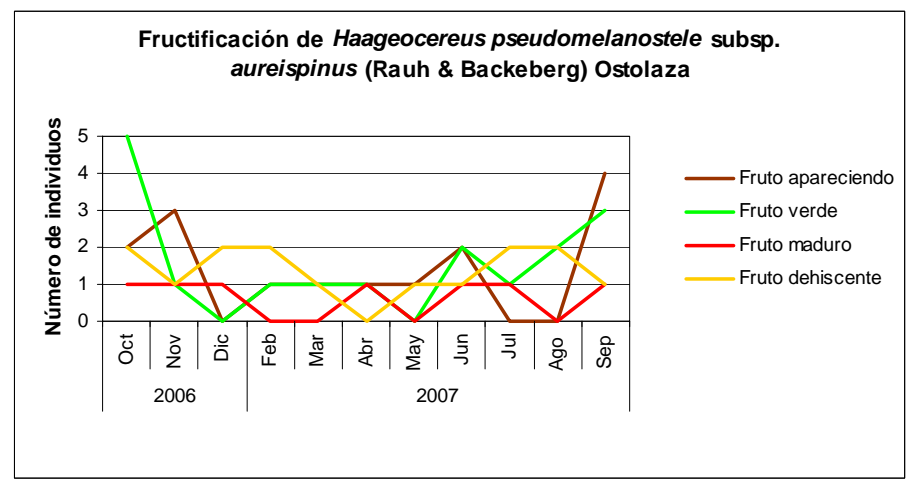

Figura 7. Fenograma de las etapas de fructificación de Haageocereus pseudomelanostele subsp. aureispinus (Rauh \& Backeberg) Ostolaza, en relación con el número de individuos Octubre 2006 - Setiembre 2007. 


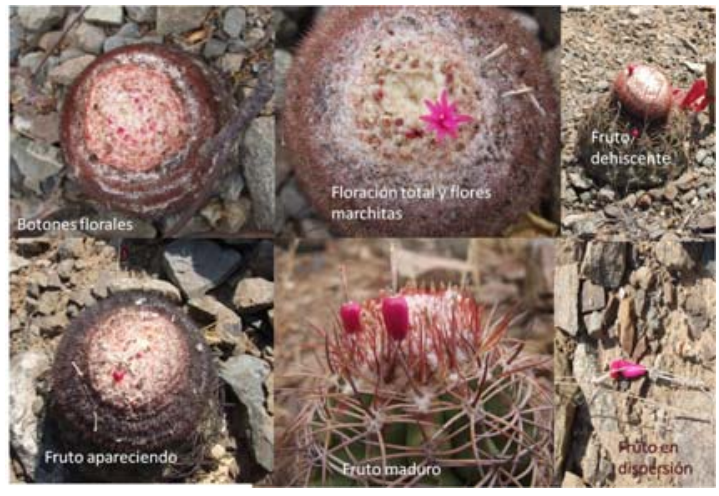

Figura 8. Estadios fenológicos para las fenofases de floración y fructificación de Melocactus peruvianus Vaupel. Cerro Umarcata. Valle del río Chillón.

embargo, la HR presentó sus mayores valores en los meses de febrero, marzo y abril lo que favoreció el desarrollo de Fruto Verde, Fruto Apareciendo y Floración Total (Figura 17).

El principal factor climático que afecta la fenología de las plantas es la temperatura y se sabe que incrementos en la temperatura del aire pueden ser detectados fácilmente en los datos fenológicos (Menzel, 2000; citado por Alvarado et al., 2002). Ruiz et al., (2000) consideró a la temperatura dentro de otras variables climáticas (precipitación, brillo solar y humedad relativa) para explicar los patrones fenológicos de cactáceas en el enclave seco de la Tatacoa (Colombia), como por ejemplo que la floración de Stenocereus griseus (Haw.) Britton \& Rose, presentó una correlación negativa y estadísticamente significativa con la humedad relativa media mensual y la de Pilosocereus sp. con la temperatura media mensual. Sin embargo, de la misma manera que Petit (2001), no encontró correlaciones estadísticamente significativas con la precipitación, entre las variaciones mensuales de floración y fructificación de las medias mensuales. Tampoco encontró una correlación estadísticamente significativa entre el número mensual de individuos en flor y fruto, y los patrones de precipitación. Sin embargo, encontró un mayor número de flores de $S$. griseus en la época seca y de Pilosocereus lanuginosus en la época de lluvias. Petit (2001) observó para S. griseus que los botones fueron afectados negativamente por la lluvia, con muchos botones abortados después de una fuerte lluvia, Ruiz et al., (2000) registró una mayor producción de frutos durante la época de lluvia para $S$. griseus. Petit (2001) afirma que es posible que la temperatura juegue un rol importante en la sincronización de la formación de flores. De la misma manera en el Cerro Umarcata el estadio Flor Marchita es el único correlacionado con la temperatura en $E$. melanostele, debido a que a temperaturas altas se registró un mayor número de individuos en esta fenofase. En la fenofase de fructificación se registró correlación en el estadio Fruto Apareciendo en E. melanostele, $H$. pseudomelanostele subsp. aureispinus y $M$. peruvianus. De esto se puede inferir que, mayores temperaturas inducen la producción de frutos en las especies señaladas en los meses de octubre, noviembre y diciembre. En los mismos meses los estadios Fruto Maduro y Fruto Dehiscente también se encuentran correlacionados con la temperatura en $N$. arequipensis subsp. roseiflora. En estas especies la producción de frutos sólo se registró en los meses de mayor temperatura. De manera similar, Laura (2007) encontró que la temperatura estaba relacionada con frutos maduros y que el número de individuos con frutos maduros era mayor cuando se registraban las temperaturas más altas, en las cactáceas de la comunidad de Atiquipa (Arequipa).

En cuanto a la correlación entre la HR y los estadios fenológicos, se encontró una clara relación entre el porcentaje de humedad relativa y la

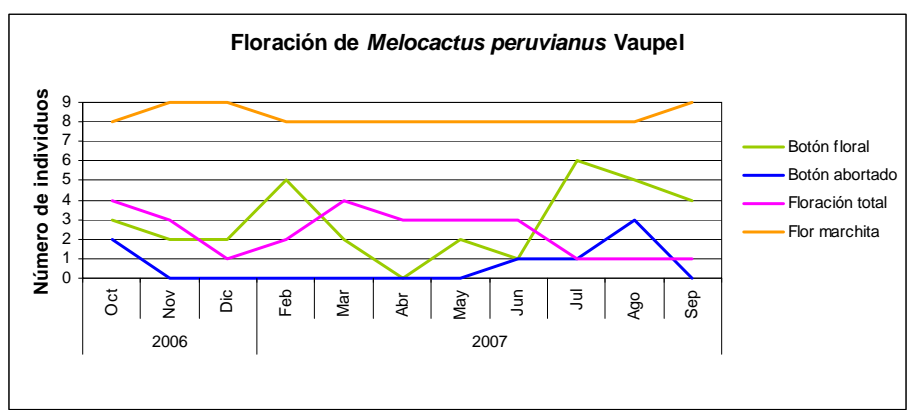

Figura 9. Fenograma de las etapas de floración de Melocactus peruvianus Vaupel, en relación con el número de individuos Octubre 2006 - Setiembre 2007.

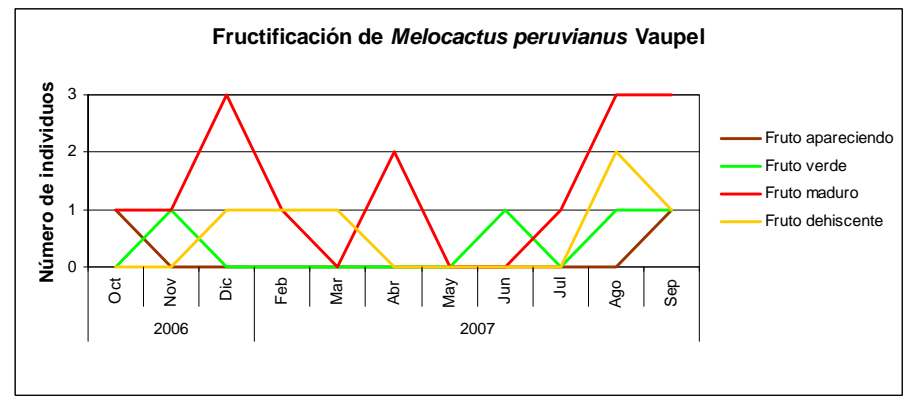

Figura 10. Fenograma de las etapas de fructificación de Melocactus peruvianus Vaupel, en relación con el número de individuos Octubre 2006 - Setiembre 2007. 


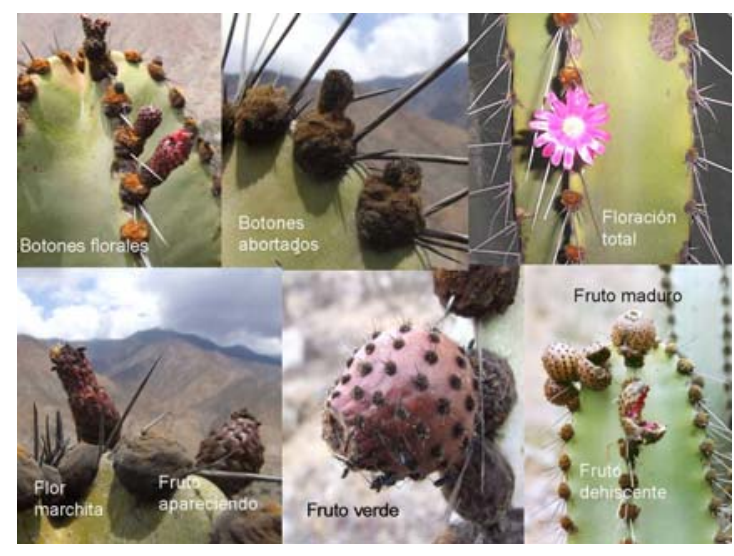

Figura 11. Estadios fenológicos para las fenofases de floración y fructificación de Neoraimondia arequipensis subsp. roseiflora (Wedermann \& Backeberg) Ostolaza Cerro Umarcata. Valle del río Chillón (Foto floración total: Inés Redolfi, Foto Fruto maduro y dehiscente: Aldo Ceroni).

abundancia del estadio Fruto Verde en $N$. arequipensis subsp. roseiflora, así como también con el estadio Fruto Apareciendo. En E. melanostele los estadios fenológicos correlacionados con la humedad relativa fueron Botón Floral y Floración Total. Al ser este un cactus que alcanza alturas de hasta $2 \mathrm{~m}$, tendrá las flores más expuestas a las condiciones del medio, por lo tanto un mayor registro de HR favorecería al buen desarrollo de sus flores. Así mismo, también se observó que en $M$. peruvianus y $N$. arequipensis subsp. roseiflora el estadio Floración Total tiene relación con el porcentaje de humedad relativa en el ambiente.

\section{Conclusiones.}

1. Se encontraron dos fenofases, floración y fructificación con ocho estadios fenológicos en Esposta melanostele, Haageocereus pseudomelanostele subsp. aureispinus, Melocactus peruvianus y Neoraimondia arequipensis subsp. roseiflora entre Octubre 2006-Setiembre 2007.

2. Para la fenofase de floración, los estadios fenológicos Flor Marchita y Botón Abortado fueron dominantes.

3. Para a la fenofase de fructificación, el estadio fenológico Fruto Maduro se presenta en un porcentaje bajo durante el año.

4. El factor Temperatura se correlacionó positivamente con los estadios fenológicos Flor Marchita, Fruto Apareciendo, Fruto Maduro y Fruto Dehiscente.

5. El factor Humedad Relativa se correlacionó positivamente con los estadios fenológicos Botón Floral, Floración Total, Fruto Apareciendo y Fruto Verde.

\section{Literatura citada.}

Alvarado M., Foroughbackhch R., Jurado \& E. Rocha A. 2002. El cambio climático y la fenología de las plantas. Ciencia UANL Vol 5. No4: 493 - 500.

Bronstein J.L. 1995. The plant-pollinator landscape. In: Hansson L.; Fahrig L. and Merriam G. (eds), Mosaic Landscapes and Ecological Processes. Chapman \& Hall, London.

Castro V. 2006. Taxonomía de la familia Cactaceae en el valle de río Chillón, Lima: cerro Umarcata y quebrada Oropel. Tesis para optar el Grado Académico de Magíster Scientiae. Universidad Nacional Agraria La Molina. 96 p.

Ceroni A., Castro V., Calderón N. \& Novoa S. 2004. Cactario del Jardín Botánico “Octavio Velarde Núñez” de la Universidad Nacional Agraria La Molina. Quepo. Vol. 18. pp. 34 - 38.

Ceroni A. \& Castro V. 2006. Los cactus del Cerro Umarcata. Lima. Canta. Perú. Revista de Biologia Augusto Weberbauer. (9): 15 - 18.

Ceroni A., Castro V., Teixeira V. \& Redolfi I. 2007.

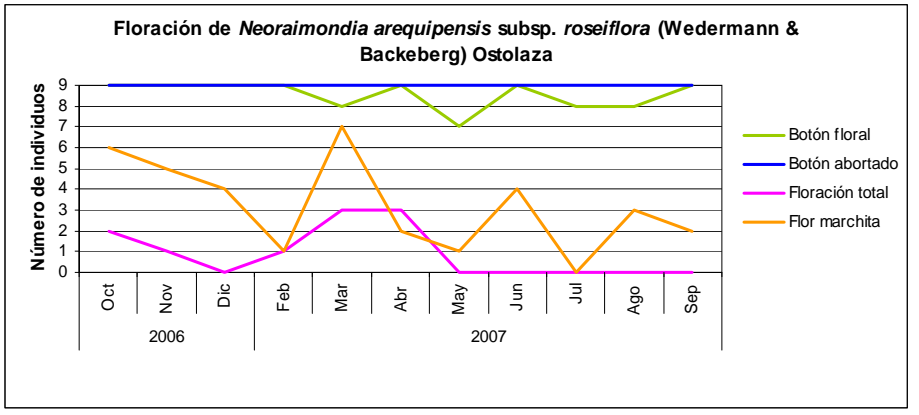

Figura 12. Fenograma de las etapas de floración de Neoraimondia arequipensis subsp. roseiflora (Wedermann \& Backeberg) Ostolaza, en relación con el número de individuos Octubre 2006 - Setiembre 2007.

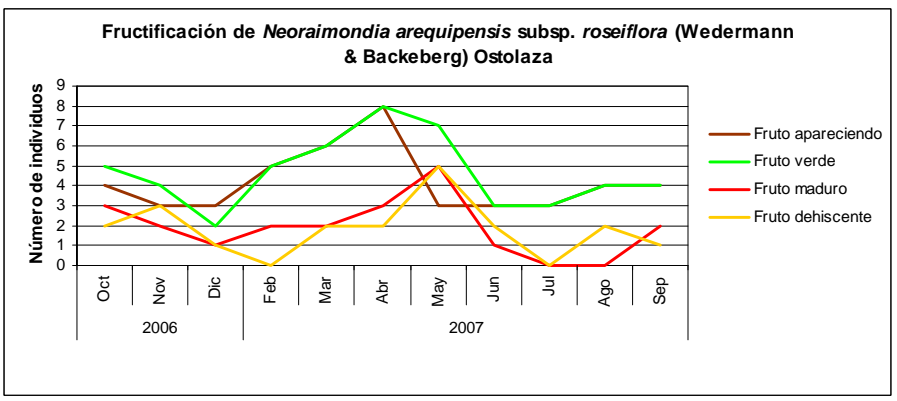

Figura 13. Fenograma de las etapas de fructificación de Neoraimondia arequipensis subsp. roseiflora (Wedermann \& Backeberg) Ostolaza, en relación con el número de individuos Octubre 2006 - Setiembre 2007. 
Neoraimondia arequipensis subsp. roseiflora (Werdermann \& Backeberg) Ostolaza (Cactaceae): eje de las interacciones en ecosistemas áridos. Ecología Aplicada. 6 (1,2): 155 - 168.

Colaço M., Fonseca R., Lambert S., Costa C., Machado C. \& Borba E. 2006. Biología reproductiva de Melocactus glaucescens Buining \& Brederoo e M. paucispinus G. Heimen \& Paul R. (Cactaceae), na Chapada Diamantina, Nordeste do Brasil. Revista Brasil. Bot. 29(2): 239 249.

De Viana M., Ortega B., Saravia M. \& Schlumpberger B. 2001. Biología floral y polinizadores de Trichocereus pasacana (Cactaceae) en el Parque Nacional Los Cardones, Argentina. Revista de Biología Tropical. 49(1): 279 - 285.

Fleming T. \& Holland J. 1998. The evolution of oblígate pollination mutualisms: senita cactus and senita moth. Oecologia. 114: 368 - 375.

Font Quer P. 2000. Fenología Diccionario de botánica. Primera Edición. Ediciones Península. Barcelona. 1280 p.

Galán C. 2006 Métodos fenológicos recientes aplicados a la Aerobiología. XV International A.P.L.E, Symposium of Palynology, Polen 16:13.

Holland J. \& Fleming T. 1999. Mutualistic interactions between Upiga virescens (Pyralidae), a pollinating seedconsumer, and Lophocereus schottii (Cactaceae). Ecology. 80(6): 2074 - 2084.

Holland J. \& Fleming T. 2002. Co-pollinators and specialization in the pollinating seed-consumer mutualism between senita cacti and senita moths. Oecología. 133: 534-540.

Ibarra-Cerdeña C., Iñiguez-Dávalos L. \& Sánchez-Cordero V. 2005. Pollination ecology of Stenocereus queretaroensis (Cactaceae) a chiropterophilous columnar cactus, in a tropical dry forest of México. American Journal of Botany. 92(3): 503-509. Keopcke H. 1973. Die Lebensformen Band I. Goecke.

Laura M. 2007. Especies, Distribución y Fenología Reproductiva de La Familia Cactaceae en la Comunidad Campesina de Atiquipa, Caravelí- Arequipa, 2005 2006 Tesis para optar el título de Biólogo. Universidad Nacional de San Agustín. Arequipa. Perú.

McIntosh M. 2002. Flowering phenology and reproductive output in two sister species of Ferocactus (Cactaceae). Plant Ecology. 159: 1-13.

Molina F., Rojas A., Fleming T. \& Valiente A. 2004. Pollination biology of the columnar cactus Pachycereus pecten-aboriginum in north-western México. Journal of Arid Environments. 56: 117-127.

Nassar J., Ramírez N. \& Linares O. 1997. Comparative pollination biology of venezuelan columnar cacti and the role of nectar-feeding bats in their sexual reproduction. American Journal of Botany. 84(8): 919-927.

Novoa S., Castro V., Ceroni A. \& Redolfi I. 2003. Relación entre la hormiga Camponotus sp. (Hymenoptera: Formicidae) y una comunidad de cactus (Cactaceae) en el valle del río Chillón. Ecología Aplicada. 2 (1): 69 73.

Novoa S., Ceroni A. \& Arellano C. 2005a. Contribución al conocimiento de la fenología del cactus Neoraimondia arequipensis subsp. roseiflora (Werdermann \&
Backeberg) Ostolaza (Cactaceae) en el valle del río Chillón, Lima-Perú. Ecología Aplicada. 4 (1 y 2): 35 40.

Novoa S., Redolfi I. \& Ceroni A. 2005b. Patrón de actividad diario de la hormiga Camponotus sp. en los botones florales del cactus Neoraimondia arequipensis subsp. roseiflora (Werdermann \& Backeberg) Ostolaza. Ecología Aplicada. 4 (1 y 2): 77 - 81.

Novoa S., Redolfi I., Ceroni A. \& Arellano C. 2005c. El forrajeo de la hormiga Camponotus sp. en los botones florales del cactus Neoraimondia arequipensis subsp. roseiflora (Werdermann \& Backeberg) Ostolaza (Cactaceae). Ecología Aplicada. 4 (1 y 2): 83 - 90.

Novoa S. 2005. Fenología del cactus Neoraimondia arequipensis ssp. roseiflora (Werdermann \& Backeberg) Ostolaza (Cactaceae) y la relación con la visita de la hormiga Camponotus sp. (Hymenoptera: Formicidae) en el valle del río Chillón, Lima-Perú. Tesis para optar el título de Biólogo Universidad Nacional Agraria La Molina. Lima .Perú.

Oficina Nacional de Evaluación de Recursos Naturales (ONERN).1976. Mapa Ecológico del Perú: Guía Explicativa. Lima, Perú.

Petit S. 2001. The reproductive phenology of three sympatric species of columnar cacti on Curacao. Journal of Arid Enviroments. 49: 521- 531.

Rojas A., Valiente A., Arizmendi M., Alcántara A. \& Arita H. 1999. Seasonal distribution of the long nosed bat (Leptonicteris curosoae) in the North America: does a generalized migration pattern really exist?. Journal of Biogeography. 26: 1065 - 1077.

Rondón J. 1994. Sinopsis de las principales metodologías aplicadas a los estudios fenológicos de los árboles tropicales. Revista Forestal Latinoamericana. 14: 5 - 32.

Rondón J. \& Pulido R. 2001. Aspectos fenológicos de las cactáceas de la zona xerófila del estado MéridaVenezuela. Revista Forestal Venezolana. 45(1): 57- 63.

Ruiz A., Santos M. \& Cavalier J. 2000. Estudio Fenológico de Cactáceas en el Enclave Seco de la Tatacoa, Colombia. Biotropica 32(3): 397- 407.

Sahley C. 1996. Bat and huming bird pollination of an autotetraploid columnar cactus, Weberbauerocereus webwerbaueri (Cactaceae). American Journal of Botany. 83(10): 1329-1336.

Teixeira V., Castro V., Ceroni A. \& Eyzaguirre R. 2004. Diversidad y densidad de la comunidad de cactáceas en el valle del río Chillón: cerro Umarcata y quebrada Orobel y su relación con los factores edáficos. Ecología Aplicada. 3 (1 y 2): 1- 8.

Valiente A., Del Coro M., Rojas A. \& Domínguez L. 1996. Ecological relationships between columnar cacti and nectar-feeding bats in México. Journal of Tropical. Ecology. 12(1): 103-119.

Valiente A., Rojas A., Del Coro M. \& Dávila P. 1997. Pollination Biology of two columnar cacti (Neubuxbaumia mezcalaensis and Neobuxbaumia macrocephala) in the Tehuacan Valley, central Mexico. American Journal of Botany. 84(4): 452 - 455.

Weberbauer A. 1945. El mundo vegetal de los Andes Peruanos: Estudio fitogeográfico. 2da Edición. Estación Experimental Agrícola de La Molina. Dirección de Agricultura. Ministerio de Agricultura. Lima. 776 pp. 


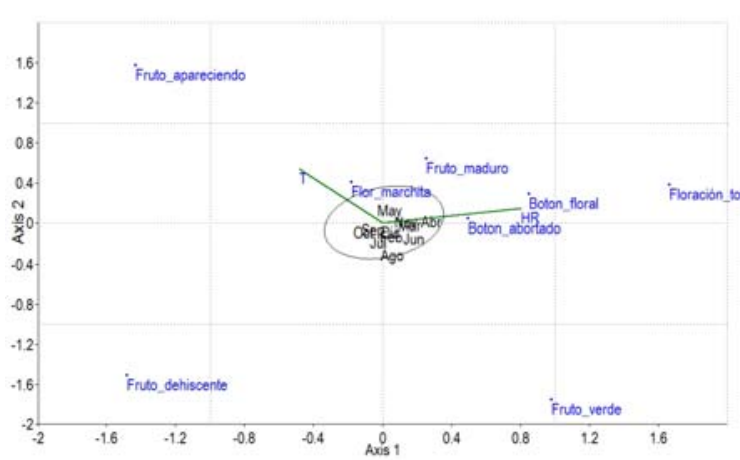

Figura 14. Análisis de correlación canónica de la fenología de Espostoa melanostele (Vaupel) Borg y los parámetros ambientales ( $\mathrm{T}$ y HR) entre los meses Octubre 2006 hasta Setiembre 2007.

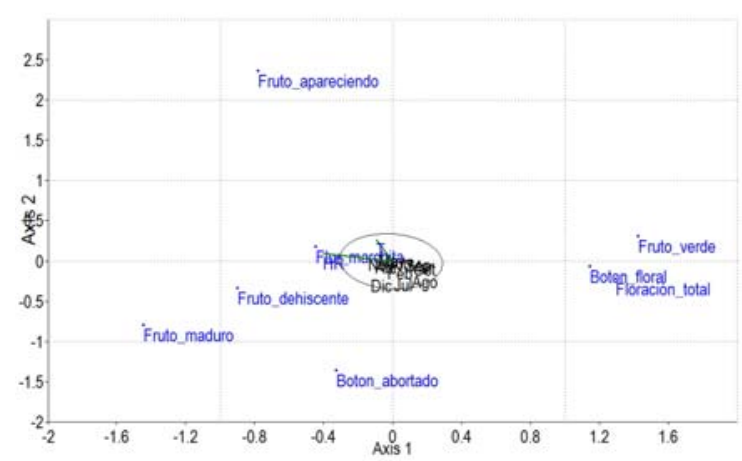

Figura 15. Análisis de correlación canónica de la fenología Haageocereus pseudomelanostele subsp. aureispinus (Rauh \& Backeberg) Ostolaza y los parámetros ambientales ( $\mathrm{T}$ y HR) entre los meses Octubre del 2006 hasta Setiembre 2007.

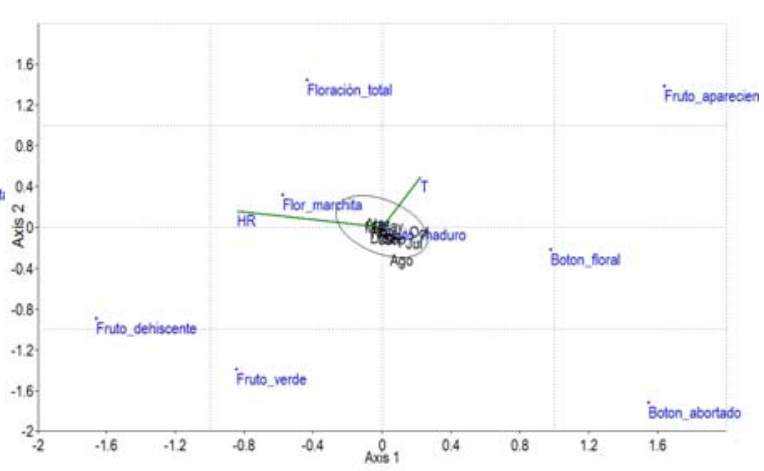

Figura 16. Análisis de correlación canónica de la fenología Melocactus peruvianus Vaupel y los parámetros ambientales ( $\mathrm{T}$ y HR) entre los meses Octubre 2006 hasta Setiembre 2007.

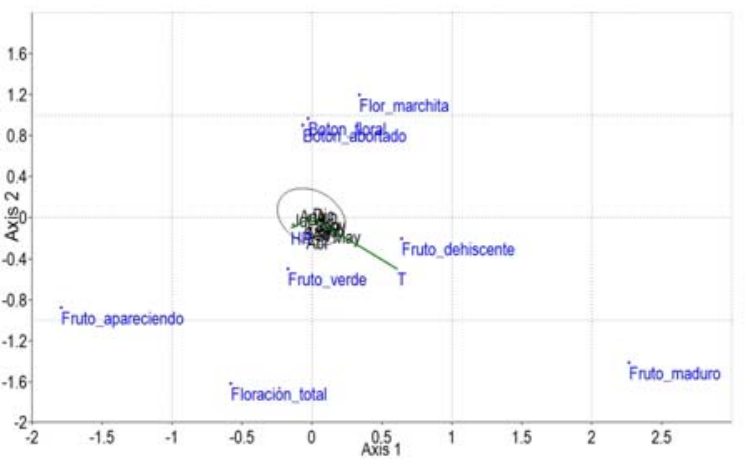

Figura 17. Análisis de correlación canónica de Neoraimondia arequipensis subsp. roseiflora (Wedermann \& Backeberg) Ostolaza entre los meses Octubre 2006 hasta Setiembre 2007.

1 Afiliación cuando se realizó la Investigación: Jardín Botánico “Octavio Velarde Núñez”. Departamento Académico de Biología, Universidad Nacional Agraria La Molina, Av. La Molina s/n, Lima - Perú. Afiliación actual: Department of Biological Science, Florida State University, USA. Correo Electrónico: nmiller@bio.fsu.edu

2 Jardín Botánico “Octavio Velarde Núñez”. Departamento Académico de Biología. Universidad Nacional Agraria La Molina. Av. La Molina s/n. Lima - Perú. aceroni@lamolina.edu.pe ; vcastro@lamolina.edu.pe 